\title{
Tetanus
}

\section{Poudel $\mathbf{P}^{1}$, Budhathoki $\mathbf{S}^{\mathbf{1}}$, Manandhar $\mathrm{S}^{\mathbf{2}}$}

${ }^{1}$ Assistant Professor, Department of Paediatrics and Adolescent Medicine, BP Koirala Institute of Health Sciences, Dharan, Nepal, ${ }^{2}$ Lecturer, Department of Paediatrics, Kathmandu Medical College, Sinamangal, Nepal.

\begin{abstract}
Tetanus is now a rare disease in developed world. However it remains an important cause of death worldwide and is associated with a high case fatality, particularly in the developing world. Tetanus is caused by contamination of wound by spores of Clostridium tetani. Neonatal tetanus results from contamination of the umbilical stump at or following delivery of a child born to a mother who did not possess sufficient circulatory antitoxin to protect the infant passively by transplacental transfer. It produces its clinical effects via a powerful exotoxin, tetanospasmin, which leads to uncontrolled disinhibited efferent discharges from motor neurons in the spinal cord and brainstem, causing intense muscular rigidity and spasm. Shorter incubation and onset times are associated with more severe disease and poorer prognosis. Four clinical forms of tetanus are recognised. They are generalised, localised, cephalic and neonatal tetanus. Tetanus is associated with several complications like respiratory failure, cardiovascular instability, renal failure and autonomic dysfunctions. Recovery from tetanus takes a long time. Diagnosis is established clinically. Symptomatic management, early recognition of complications, careful monitoring for dysautonomia and respiratory assistance are the anchors for successful outcome of patients. Tetanus is preventable through vaccination. Vaccination is highly safe and efficacious. Active immunisation should be instituted in all partially immunised, unimmunised persons and those recovering from tetanus. Passive immunisation is given as treatment of a case as well as prevention following high risk injury. Nepal has achieved neonatal tetanus elimination status on 2005 and is running different programs to sustain the status.
\end{abstract}

Key words: Tetanus, neonatal tetanus, spasm, treatment, immunisation.

$\mathrm{T}$ tanus was described by Hippocrates approximately 30 centuries ago $^{1}$. In 1884 Nicolaier produced tetanus in animals by injecting them with soil specimen ${ }^{2}$. Kitasato isolated the organism in 1889 from a human tetanus victim and reported neutralisation of toxin by specific antibodies ${ }^{2}$. Tetanus toxoid was described by Descombey in 1924 and was effectively used during the world war $\mathrm{II}^{2}$. Tetanus and neonatal tetanus (NT) used to be a major cause of morbidity and mortality in Nepal. To control tetanus government of Nepal has been launching different programs since past. Since the introduction of Expanded Program on Immunisation (EPI) in 1979, it has significantly contributed to reduce the burden of vaccine preventable diseases including tetanus and $\mathrm{NT}^{3}$. Different programs like EPI, maternal and neonatal tetanus elimination program, safe motherhood program, tetanus toxoid (TT) campaign, training of birth attendants, health education etc were the milestones for achieving control over this disease in $\mathrm{Nepal}^{3}$. Due to all these efforts, NT has been eliminated in Nepal since $2005^{3}$. NT elimination status is the incidence of NT less than one case per 1000 live births ${ }^{3}$. Now Nepal is aiming at controlling tetanus and sustaining NT elimination status ${ }^{3}$. To achieve this aim,
Nepal government, in addition to continuing routine programs of past, has also recently introduced programs like integrated vaccine preventable diseases surveillance, school immunisation against tetanus, community based maternal neonatal care and safe delivery incentives ${ }^{3}$. In fiscal year 2006/2007, school immunisation against tetanus was implemented in 11 districts and community based maternal neonatal care program in three districts, two doses of TT were given to 546646 women of child bearing age covering $50.0 \%$ of eligible women and three doses of Diphtheria, Polio, and Tetanus (DPT) were given to $84.0 \%$ of eligible children ${ }^{3}$. In addition to two doses of TT to pregnant women, three doses of TT to students of grades one, two and three has been also included in EPI of Nepal since fiscal year 2006/2007³.

\section{Problem Statement}

Tetanus remains an important cause of death and is associated with a high case fatality, particularly in the

Correspondence

Dr. Prakash Poudel

Department of Paediatrics and Adolescent Medicine

BP Koirala Institute of Health Sciences, Dharan, Nepal.

E-mail: prakashpdl@hotmail.com 
developing world ${ }^{2}$. The global incidence of tetanus is about 18 cases per 100000 population per year with case fatality ranging between $20.0 \%$ to $50.0 \%{ }^{2}$. In industrialised countries, tetanus has been virtually eliminated. USA reported 43 cases per year from 1998 to $2000^{4}$. In 1996, estimated deaths from neonatal tetanus (NT) were 438080 with mortality rates of 4.8 to 10.7 per 1000 live births in high risk developing countries ${ }^{5}$. In Nepal, there were 6700 estimated deaths due to NT with mortality rate of 8.8 per 1000 live births in the year $1990^{6}$. In the year 1997, estimated deaths due to NT declined to 5800 with mortality of seven per 1000 live births ${ }^{6}$. Tetanus cases are probably under reported in Nepal. During the fiscal year 2003/2004, 63 cases of NT and 169 cases of non neonatal tetanus were reported ${ }^{7}$. During the fiscal year 2006/2007, 42 cases of NT and 155 cases of non neonatal tetanus were reported ${ }^{3}$. In BP Koirala Institute of Health Sciences (BPKIHS), 282 cases were treated for tetanus between 1993 and $2003^{8}$. In another study conducted at BPKIHS over a 22 months period from 2004 to 2006, there were 19 cases of pediatric tetanus (1-14 years age) and five cases of $\mathrm{NT}^{9}$. Beyond neonatal period, tetanus is uncommon in pediatric population as compared to adults. In study by Lau et al, only $1.0 \%$ cases were below 20 year age ${ }^{10}$. During the fiscal year 2006/2007 in Nepal, among total inpatients morbidity due to non NT, highest number of cases were between the age group of 20 to 49 years accounting for $43.2 \%$ of cases $^{3}$. Paediatric tetanus among non NT accounted for $26.3 \%$ cases $^{3}$.

\section{Microbiology}

Tetanus is caused by Clostridium tetani, a gram positive, motile, obligate anaerobe, spore forming bacillus which give it a characteristic drumstick or tennis racket appearance ${ }^{1}$. This is a ubiquitous bacterium with a natural habitat of soil, but can also be isolated from stools of domestic animals and nearly $10.0 \%$ of humans $^{2}$. It is rarely cultured ${ }^{11}$. It produces its clinical effects via a powerful exotoxin-Tetanospasmin ${ }^{1}$.

\section{Mode of Transmission}

Tetanus is caused by contamination of wound by spores of C. tetani. It is not transmitted from person to person ${ }^{12}$. Tetanus occurs as a complication of puncture wounds, abrasions, burns, injections, surgery, compound fracture, animal bites or scratches, gastrointestinal infections, abortion or childbirth ${ }^{12}$. In $15.0 \%$ to $25.0 \%$ of patients there is no evidence of recent wound ${ }^{11}$. In study by Patel et al in 8697 tetanus cases, $29.4 \%$ cases were because of otitis and $18.1 \%$ cases had unidentified injury ${ }^{13}$. Eman et al found $36.4 \%$ cases to be injection drug users ${ }^{14}$. NT results from contamination of the umbilical stump due to unclean delivery or traditional application of contaminated material at or following delivery of a child born to a mother who did not possess sufficient circulatory antitoxin to protect the infant passively by transplacental transfer ${ }^{12,15}$. In a study done at BPKIHS on paediatric tetanus and NT, all cases of NT were because of umbilical sepsis and major causes of non NT were lacerated wound $(32.0 \%)$, otitis media $(16.0 \%)$ and unnoticed minor injury $(16.0 \%)^{9}$.

\section{Pathogenesis}

The tetanus bacillus secretes two toxins: tetanospasmin and tetanolysin. Tetanolysin locally damages tissue and optimises the conditions for bacterial multiplication ${ }^{11}$. Tetanospasmin leads to clinical syndrome of tetanus. Released tetanospasmin spreads to underlying tissue and binds to gangliosides GD18 and GT1b on the membranes of local nerve terminals. Some amount may enter blood stream from where it diffuses to bind nerve terminals throughout the body. The toxin is then internalised and transported intraaxonally and retrogradely to the cell body, from where the toxin can diffuse out, affecting and entering nearby neurons ${ }^{11}$. The symptoms appear when spinal inhibitory neurons are affected ${ }^{16}$. The effects of the toxin result from the prevention of release of neurotransmitters ${ }^{11}$. The toxin has a predominant effect on inhibitory neurons ${ }^{11}$. Interneurons inhibiting alpha motor neurons are first affected and the motor neurons lose inhibitory control. Later, because of the longer path, preganglionic sympathetic neurons and the parasympathetic centres are also affected. Tetanospasmin has a cortical convulsant effect in animal models ${ }^{17}$. The prejunctional effect on neuromuscular junction may lead to considerable weakness between the spasms and might account for both the paralysis of cranial nerves observed in cephalic tetanus and myopathies observed after recovery ${ }^{18}$. Uncontrolled disinhibited efferent discharges from motor neurons in the cord and brainstem lead to muscular rigidity and spasm, which may mimic convulsions. Muscles of the jaw, face and head are often involved first because of their shorter axonal pathways. The trunks and limbs follow but muscles in the hand and feet are relatively spared ${ }^{11}$. Disinhibited autonomic discharges lead to disturbance in autonomic control with sympathetic over activity ${ }^{11}$. Neuronal binding of toxin is irreversible. Recovery requires the growth of new nerve terminals, which explains the prolonged course of tetanus ${ }^{16}$.

\section{The incubation period and onset time}

The incubation period in tetanus averages seven to ten days with a range of one to sixty days ${ }^{11}$. Incubation period may be related to the amount of toxin and immunisation status of the patient ${ }^{2}$. Saltoglu et al found mean incubation period as 11.5 days with a range of two to forty days ${ }^{19}$. The usual incubation period in NT is five to ten days, thus most cases of NT have their onset in the later part of first week or early in the second week of $\operatorname{life}^{20}$. Since almost all NT cases result from 
umbilical sepsis acquired at or shortly after birth, age of presentation matches the incubation period. The onset time is the time from first symptom to the first $\operatorname{spasm}^{1,2,11}$. It varies from one to seven days ${ }^{11}$. Shorter incubation and onset times are associated with more severe disease ${ }^{2,11}$. In $10.0 \%$ to $30.0 \%$ of cases, tetanus follows unrecognised minor injury and the incubation period is therefore uncertain ${ }^{1}$. In a study at BPKIHS on paediatric and NT, mean incubation period was 7.7 days with a range of two to 17 days $^{9}$. Mean onset time was 16.9 hours with a range of two to 72 hours 9 .

\section{Clinical Features}

Four clinical forms of tetanus are recognised ${ }^{2}$.

Generalised tetanus:- This is the commonest form and is characterised by increased muscle tone and generalised spasms. Usually the first symptom is difficulty in opening jaw - trismus or lock jaw which occurs in up to $93.0 \%$ of case ${ }^{21}$. Some cases may initially present with trismus alone $^{19}$. Subsequently spasm spread to other body parts and patient develops dysphagia, rigidity and pain in neck, abdomen and back, recurrent muscle spasms and dysarthria $^{21}$. Facial grimacing popularly known as 'risus sardonicus' (ironical smile), may appear due to facial muscle contraction. Eventually generalised muscle spasms occur spontaneously or due to minor stimuli such as noise, touch or nursing procedures, which causes ophisthotonus ${ }^{2}$. The spasms are painful and may lead to respiratory compromise. Autonomic instability is of major concern and usually develops few days after the onset of disease ${ }^{2}$. There may be labile or sustained hypertension, peripheral vasoconstriction, hypotension, tachycardia, bradycardia, arrhythmia, recurrent cardiac arrest and hyperpyrexia without secondary infection ${ }^{2,11}$. Other autonomic effects include profuse salivation, increased bronchial secretions, gastric stasis, ileus and high output renal failure ${ }^{11}$. Juma et al found autonomic dysfunction in $100.0 \%$ of their patients including NT whereas Wasay et al found autonomic dysfunction in only $32.0 \%$ of the patients ${ }^{21,22}$.

Neonatal tetanus:-The common age of onset is between five to fifteen days following birth ${ }^{2}$. Common presenting features are rigidity, spasms, failure to suck, trismus, fever and seisures ${ }^{2,20}$. Additional features may be vomiting, cyanosis, flexed toes and ophisthotonus ${ }^{11,20}$.

Localised tetanus: This is uncommon and benign form of the disease in which patients have persistent contraction of muscles in same anatomic area as the injury preceding the tetanus ${ }^{1,2}$. Localised tetanus might generalise later ${ }^{1,2}$. Partial immunity may be responsible for the localised form of tetanus ${ }^{1}$.

Cephalic tetanus: This is a form of localised tetanus restricted to head and neck, with a high potential to generalise later ${ }^{11}$. It commonly results from middle ear infection and head injury ${ }^{2}$. Facial muscles are most commonly affected followed by sixth, third, fourth and twelfth cranial nerves in the order of frequency ${ }^{2}$. Trismus may be present but follows other cranial nerve deficits in $42.0 \%$ of patients ${ }^{23}$.

In a study at BPKIHS in neonates and children, generalised tetanus accounted for $75.0 \%$ cases and $20.8 \%$ cases were $\mathrm{NT}^{9}$. A child initially presented as cephalic tetanus later progressed to generalised tetanus ${ }^{9}$. Generalised spasm was the presenting feature in $94.7 \%$ children and $60.0 \%$ neonates 9 . Autonomic dysfunctions occurred in $96.0 \%$ casses ${ }^{9}$. Common autonomic dysfunctions were fever (83.3\%), tachycardia (79.2\%), hypotension (45.8\%) and hypertension $(25.0 \%)^{9}$.

\section{Severity Grading}

Several grading systems are reported (Philips, Dakar, Udwadia etc) ${ }^{11}$. The system reported by Ablett is most widely used (Table 1). ${ }^{24}$

Table 1: Ablett classification of severity of Tetanus

\begin{tabular}{|l|l|l|}
\hline \multicolumn{2}{|l|}{ Grade } & Clinical features \\
\hline I & Mild & $\begin{array}{l}\text { Mild to moderate trismus, generalised spasticity, no respiratory embarrassment, no spasms, } \\
\text { little or no dysphagia. }\end{array}$ \\
\hline II & Moderate & $\begin{array}{l}\text { Moderate trismus, marked rigidity, mild to moderate but short spasms, moderate respiratory } \\
\text { embarrassment with an increased respiratory rate }(>30), \text { mild dysphagia. }\end{array}$ \\
\hline III & Severe & $\begin{array}{l}\text { Severe trismus, generalised spasticity, reflex prolonged spasms, RR }>40, \text { apnoeic spells, } \\
\text { severe dysphagia, tachycardia( heart rate }>120)\end{array}$ \\
\hline IV & Very severe & $\begin{array}{l}\text { Grade III and violent autonomic disturbances involving the cardiovascular system. Severe } \\
\text { hypertension and tachycardia alternating with relative hypotension and bradycardia, either } \\
\text { of which may be persistent. }\end{array}$ \\
\hline
\end{tabular}




\section{Complications}

Complications result from the disease itself or are related to management ${ }^{19}$.

Respiratory complications are bronchopulmonary infections, atelectasis, iatrogenic pneumothorax, complications related to tracheotomy (e.g. bleeding), adult respiratory distress syndrome, apnoeic spells and respiratory failure. The cardiovascular and autonomic complicationsincludesustainedtachycardia,hypotension, hypertension, paroxysmal supraventricular tachycardia, ventricular extra systoles, sudden cardiac arrest, heart block, pulmonary embolism, hyperpyrexia and profuse sweating. Gastrointestinal (GI) complications include GI bleeding, ileus and diarrhoea. Renal complications are renal failure due to rhabdomyolysis or drugs, prerenal and mixed renal failure. Hypokalaemia, hyperkalaemia, hyponatremia are common electrolyte disturbances. Central nervous system (CNS) complications include unexplained coma, seizure and peripheral neuropathy. Other complications are septic shock, disseminated intravascular coagulation, thrombophlebitis, urinary tract infections, bed sores, dry eyes and fractures.

In a study at BPKIHS on paediatric and NT, major complications noted were respiratory failure $(45.8 \%)$, pneumonia (37.5\%), Myoglobinuria (37.5\%) and hypoxic encephalopathy $(25.0 \%)^{9}$.

\section{Natural history}

In the first week, there is increase in severity of spasms. Autonomic disturbances usually start several days after spasms and persist for one to two weeks. Spasms reduce after two to three weeks, but stiffness may persist longer ${ }^{11,16}$. Recovery is expected to be complete. However, in one of the follow up studies in survivors of tetanus, persisting physical and psychological problems were frequent ${ }^{25}$. Recovery from tetanus takes a long time. In a study, patients required 11 to 30 days stay in hospital $^{10}$. In another study, mean duration of hospital stay was 5.5 weeks (range five to seven weeks) ${ }^{22}$. Localised tetanus may recover or may generalise over time $^{2}$. Cephalic tetanus course ranges from mild to severe disease with multiple cranial nerve involvement and high mortality ${ }^{2}$.

In NT, due to lack of inhibiting influences from higher centres in newborns, they have more severe spasms ${ }^{2}$. In few studies mean age of onset ranged from 3.6 to 6.3 days and mean age of death from 7.9 to 10.3 days, with the mean interval between onset and death between two to three days ${ }^{26}$. Bwire et al found mean age at onset 5.6 days with mean age at death 9.9 days ${ }^{26}$. Jeena et al found mean duration of ICU stay 35 days (range: 13 to 87 days $)^{15}$. One case reported from USA from 1998 to 2000 required hospital admission for 19 days $^{4}$.
In a study at BPKIHS on paediatric and NT, the mean duration of hospital stay among survivors was 21.5 days with a range of 14-28 days ${ }^{9}$. Mean interval of death was 3.75 days from admission 9 .

\section{Diagnosis and differential diagnosis}

Diagnosis is established clinically ${ }^{27}$. No definite laboratory abnormalities are present. Cerebro-spinal fluid is usually normal ${ }^{2}$. C. tetani is usually not visible on gram stain of wound materials ${ }^{27}$. Cultures of umbilical stump are usually negative ${ }^{20}$.

Differential diagnosis of tetanus includes painful conditions of lower jaw, Bell's palsy, meningitis, drug induced dystonia and dyskinesia, rabies, globus hystericus, strychnine poisoning, tetany, acute abdomen, stiffiman's syndrome etc ${ }^{2}$.

Apte and Karnad described a bedside 'spatula test' to aid in diagnosis with a sensitivity of $94.0 \%$ and specificity of $100.0 \%{ }^{2}$. A spatula is inserted into the pharynx. If patient gags and expels the spatula the test is negative for tetanus. If the patient bites the spatula because of the reflex spasm of masseter, the test is considered positive ${ }^{2}$.

\section{Management}

Treatment strategies involve three principles: organisms in the body should be destroyed to prevent further toxin release, toxin outside the CNS should be neutralised, and the effects of toxin already bound to CNS should be minimised. Symptomatic management, early recognition of complications, careful monitoring for dysautonomia and respiratory assistance are the anchors for successful outcome of patients ${ }^{2}$.

(a) Halting the production of toxin: Where present, wounds should be surgically debrided ${ }^{11}$. Penicillin and Metronidazole are the two major antibiotics used routinely. Penicillin is a gamma amino butyric acid (GABA) antagonist and is associated with convulsion ${ }^{11}$. Metronidazole is now being considered as drug of choice $^{2,11}$. Antibiotic is given for seven to ten days ${ }^{2}$.

(b) Neutralisation of unbound toxin: This is achieved through passive immunisation with either human or equine tetanus immunoglobulin, as early as possible because the toxin becomes inaccessible once it is bound to the nerve terminus. Human Tetanus Immunoglobulin (HTIG) is the preferred one because of lack of immunogenecity and a long half life of 24 to 31 days as compared to equine formulation which has a high likelihood of anaphylactic reactions and a half life of only two days ${ }^{2}$. The dose of equine formulation is 500 to1000 international unit (IU) per kilogram given intravenous (IV) or intramuscular (IM). The adult dose 
of HTIG is 3000 to $6000 \mathrm{IU}_{\text {given }} \mathrm{IM}^{11}$. Local infiltration around the wound is not recommended ${ }^{2}$. Dose for prophylaxis is 1500 to $3000 \mathrm{IU}$ of equine or 250 to 500 IU of human preparation ${ }^{2}$. The blood concentration of antitoxin for protection against tetanus is 0.1 unit per millilitre ${ }^{2}$. A meta-analysis showed no obvious benefit of intrathecal antitoxin ${ }^{2}$.

(c) Control of rigidity and spasms: Unnecessary stimulation should be avoided. The mainstay of treatment is sedation with a benzodiazepine. Diazepam is most commonly used. The doses used have been variable, depending upon the patient's condition and response. The average starting adult dose is 10 to 30 milligram (mg) six to eight hourly. Doses up to $120 \mathrm{mg} /$ $\mathrm{kg}$ /day have been used ${ }^{2,22}$. Diazepam reduces anxiety, induces amnesia, sedation and muscle relaxation as well as being anticonvulsant ${ }^{22}$. At high doses, respiratory depression is major problem and ventilatory assistance is necessary ${ }^{2}$. Midazolam can be used but it is costly and needs more respiratory assistance. Benzodiazepine should be tapered gradually to avoid withdrawal reactions ${ }^{2}$. Additional sedation may be provided by Phenobarbitone and Chlorpromazine ${ }^{11}$. When sedation alone is not adequate, neuromuscular blocking agents and positive pressure ventilation may be required for a prolonged period $^{11}$.

Sedation with Propofol allows control of spasms and rigidity without using neuromuscular blocking drugs ${ }^{28}$. However, prolonged use of Propofol ( $>48 \mathrm{hrs}$ ) has been associated with lactic acidosis, bradycardia and lipemia in children and is therefore not recommended for paediatric use $^{2}$. Intrathecal Baclofen, a GABA $B$ agonist, has been reported in several small series with varying success ${ }^{2,11,29}$. However, Baclofen has a narrow therapeutic range, large interindividual pharmacodynamic variability, costly and needs surgical expertise $^{2}$

Role of Magnesium sulphate in the management of tetanus has been postulated by many authors. This drug controls the spasm and dysautonomia ${ }^{2,30}$. It is usually given as a loading dose of five grams over 20 minutes followed by regular infusion of two grams per hour in adult $^{2}$. Ventilatory support must be available ${ }^{11}$. More trials and experiences for using this drug in children are required $^{2,11}$.

(d) Control of autonomic dysfunction: Different approaches to the treatment of dysautonomia have been reported as case reports or small series. There is a lack of comparative or controlled studies ${ }^{11}$. Sedation is often the first treatment. Benzodiazepines, anticonvulsants, and particularly morphine are frequently used ${ }^{11}$. Morphine is beneficial as cardiovascular stability may occur without cardiac compromise ${ }^{31}$. Chlorpromazine is also a useful sedative. Its anticholinergic and alpha adrenergic antagonism may contribute to cardiovascular stability ${ }^{11,30}$. In recent years, the short acting beta blocker, Esmolol, has been used successfully ${ }^{11}$. Isolated use of long acting beta adrenergic blocker is not recommended, because sudden cardiac death due to loss of sympathetic drive is a feature of severe tetanus ${ }^{11}$. Dolar reported the successful management of autonomic disturbance with IV atropine ${ }^{32}$. The alpha- 2 adrenergic agonist, Clonidine, has been used with variable success ${ }^{33}$.

Magnesium sulphate is a promising drug and has been used in management of spasms as well as autonomic overactivity ${ }^{2,11,30}$. Magnesium causes presynaptic neuromuscular blockade, blocks release of catecholamines, reduces receptor sensitivity to catecholamines and has anticonvulsant and vasodilator properties ${ }^{34}$. However this drug is associated with sedation, impairment of tidal volume, cough and need for ventilation at high doses $\mathrm{s}^{30,34}$. Tracheostomy and availability of ventilatory support is imperative $\mathrm{e}^{34}$. In overdose, it may cause weakness, paralysis, hypotension and bradyarrhythmia ${ }^{11}$. Attygalle et al reported that serum magnesium concentrations were predictable and readily kept within the therapeutic range by monitoring the presence of patellar tendon reflex ${ }^{11}$. More work is necessary on the role of magnesium in the routine management of severe tetanus ${ }^{11}$.

The drugs that show potential for use in future for control of dysautonomia are Sodium valproate, Acetylcholine esterase inhibitors, Dexmedetomidine and Adenosine ${ }^{11}$.

(e) Supportive and intensive care: Supportive care plays a major role in the outcome. Nutrition should be established early. Enteral nutrition is associated with a lower incidence of complications and is cheaper than parenteral nutrition. Percutaneous gastrostomy may avoid the complications of nasogastric tube feeding ${ }^{11}$. Securing the airway helps in preventing aspiration and assisting ventilation. Tracheostomy is better than endotracheal intubation because of need for prolonged mechanical ventilation ${ }^{2}$. This also avoids undue stimulation of upper airway and prevents spasms and respiratory complications from aspiration and hypoventilation ${ }^{2}$. Assisted ventilation is used when spasms impair respiration ${ }^{2}$. Weaning should be done early to minimize the problems associated with prolonged paralysis and neuromuscular blockade. Chest physiotherapy and regular tracheal suctioning are essential ${ }^{34}$. Secretion and salivation can be minimised by using Ipratropium nebulisation ${ }^{2}$. Adequate sedation is mandatory before any interventions in patients at risk of uncontrolled spasms. Other supportive measures like mouth care, nursing the patient in $15^{\circ}$ to $30^{\circ}$ sitting position, keeping intravenous access sites clean, prophylaxis 
of GI haemorrhage, prevention and management of pressure sores, psychological support and counselling etc. are significant steps ${ }^{2}$. Maintenance of temperature, blood sugar, electrolytes and intravascular volume are important steps in supportive care of NT. Excessive use of muscle relaxants may induce iatrogenic apnoea in neonates which should be regularly monitored.

Management of tetanus in resource poor settings like Nepal is a challenge because of reasons like unavailability of ICU care and ventilator when required, poor affordability of patients, high cost involved in prolonged hospital stay and large amounts of sedative drugs 9 . For example, in a study at BPKIHS, $52.6 \%$ cases left against medical advice mainly due to poor affordability 9 .

\section{Prognosis and Mortality}

The quality of supportive care determines the outcome. For non NT, according to small studies, the mortalities were $41.0 \%$ in India $^{13}, 18.2 \%$ in Malaysia ${ }^{10}, 18.0 \%$ in $\mathrm{USA}^{4}$ and $10.0 \%$ in Saudi Arabia ${ }^{14}$. This shows the difference in mortality between developing and developed countries and hence the role of quality of care in outcome. Short incubation and short onset times carry a bad prognosis ${ }^{2,11,19}$. In one study, incubation period less than seven days was significantly associated with higher mortality $(\mathrm{p}=0.07)^{19}$. In another study, onset time less than 24 hours was associated with $90.0 \%$ mortality and onset time greater than 96 hours was associated with $1.0 \%$ mortality $^{13}$. The severity, type of tetanus and age of the patient also determine the outcome $\mathrm{e}^{2,13,19}$. Severe tetanus has been found to have higher mortality ${ }^{19}$. Saltoglu et al found $92.0 \%$ mortality among severe tetanus and $58.0 \%$ mortality among moderate tetanus ${ }^{19}$. NT has higher mortality - almost $85.0 \%$ in Indian study ${ }^{13}$. Localised tetanus without secondary generalisation has only $1.0 \%$ mortality $^{2}$. Progression to generalisation worsens the prognosis ${ }^{2}$. Cephalic tetanus, although a form of localised tetanus, carries a bad prognosis and has mortality of $15.0-30.0 \% \%^{2}$. Wasay et al found that the autonomic dysfunction, irrespective of other factors, predicted the poor outcome ${ }^{21}$. Respiratory failure used to be the commonest cause of death previously ${ }^{11,13}$. With the development of intensive care and control of respiration by artificial ventilation, other causes like cardiac arrhythmia, hyperpyrexia, paralytic ileus, septic shock, nosocomial infections, and renal failure are now responsible for death ${ }^{10,11,13}$. Autonomic disturbance is the most common cause of death. In one recent study, the major cause of death was cardiac arrhythmia (54.0\% of deaths) ${ }^{21}$. Due to lack of adequate ICU facilities for management of respiratory failure, respiratory failure remains the main cause of death due to tetanus in our settings? ${ }^{9}$. In a study at BPKIHS, major cause of death was respiratory failure and mortality rates were $40.0 \%$ and $26.3 \%$ for NT and paediatric tetanus respectively 9 Overall mortality due to tetanus in BPKIHS over a period of ten years (1993-2003) was 34.8\% ${ }^{8}$. Regarding mortality of tetanus cases in Nepal, in the fiscal year $2006 / 2007$ case fatality rate was $21.2 \%$ for non NT.

\section{Prevention}

Infection does not confer immunity. Prevention is through vaccination which is highly safe and efficacious. Active immunisation should be instituted in all partially immunised, unimmunised persons and those recovering from tetanus. Vaccination is instituted in the form of a toxoid, available as a single antigen preparationtetanus toxoid (TT), combined with Diphtheria vaccine as paediatric DT or adult Td, and with both diphtheria and pertusis vaccine as DPT and DTaP (containing acellular pertusis component) ${ }^{2}$. Children under seven years should receive DPT or DTaP or paediatric DT and children above seven years and adults should receive Td formulation. Single antigen TT is not recommend ${ }^{2}$. In children, three doses of DPT vaccine are given at an interval of four to eight weeks, starting at six weeks of $\mathrm{age}^{2}$. This should be followed by booster at 18 months. The second booster is given at five to six years and third up to ten years ${ }^{2}$. The initial series for adults involves three doses. The first two doses are given four to eight weeks apart and the third is given six months after the second $^{2}$. Booster doses are required every ten years ${ }^{2}$.

Immunisation of all pregnant women is important in preventing neonatal and maternal tetanus. In unimmunised pregnant women two doses of TT should be given, the first as early as possible during pregnancy and the second at least a month later and at least three weeks before delivery ${ }^{6}$. No pregnant woman should be denied even one dose of TT if she is seen late in pregnancy ${ }^{6}$. One booster dose will be sufficient for a woman who has been immunised earlier. When such a booster is given, it will cover for subsequent pregnancies during the next five years ${ }^{6}$. Hygienic practices during childbirth are important for prevention of NT. Over the last decade, most programs in developing countries have concentrated on training the traditional birth attendants, providing home delivery kits and educating pregnant women about the clean delivery practices. Training of birth attendants alone can reduce death due to neonatal tetanus by $90.0 \%$.

In tetanus patients, passive immunisation is usually combined with active vaccination, so called activepassive immunisation ${ }^{34}$. Different sites should be chosen for immunoglobulin and toxoid injection to prevent interaction ${ }^{2}$. Prevention of tetanus after injury is a part of tetanus prevention (Table 2$)^{4}$. All wounds must be thoroughly cleaned soon after injury and surgically debrided to remove foreign bodies, soil, dust and 
necrotic tissues. Specific measures depend on the nature of the injury and immunisation status of the patient ${ }^{4}$.

The targets of second long term plan (1997-2017 AD) of Nepal are to decrease infant mortality rate to 34.4 per 1000 live births, to decrease maternal mortality ratio from 281 (2006 AD) to 134 per 100000 live births and to decrease neonatal mortality rate from 33 (2006 AD) to 15 per 1000 live births ${ }^{3}$. To improve child survival is also a millennium development goal. All these targets cannot be met without controlling and preventing tetanus in children, mothers and neonates. Nepal is on the track to achieve these goals. In order to achieve the goals, it is necessary to improve DPT3 immunisation coverage in children, TT2 immunisation coverage in women of child bearing age, increase the deliveries conducted by health personnel or trained birth attendant, increase institutional delivery, increase public awareness and train birth attendants. In the fiscal year 2006/2007, DPT3 coverage was 84.0\%, $50 \%$ women of childbearing age received TT2, 29.7\% deliveries were conducted by health workers and $15.3 \%$ of total deliveries were institutional ${ }^{3}$. Though DPT3 and TT2 coverage were $9.0 \%$ and $1.0 \%$ respectively less than those in previous fiscal year, other parameters are showing steady improvement. Deliveries conducted by health workers increased by $6.0 \%$ and institutional delivery increased by $2.0 \%{ }^{3}$. Nepal government has already completed rounds of TT campaign targeted at women aged 10-39 years ${ }^{7}$. Nepal government has now initiated school immunisation program and it has been expanded in phase wise manner to 12 districts in fiscal year $2006 / 2007^{3}$. School immunisation program has been integrated into EPI, in which TT booster doses are given to all students of grade one, two and three ${ }^{3}$.
The service is also offered to children of the same age group (six to eight years) not attending school living in surrounding areas ${ }^{3}$. Government of Nepal is also strengthening and expanding integrated surveillance of vaccine preventable diseases which also includes $\mathrm{NT}^{3}$. In order to sustain NT elimination status, safe delivery practice is a must. To encourage safe delivery at institutions, Nepal government is also providing safe delivery incentives to women who deliver in health institution ${ }^{3}$. To ensure safe delivery at home, birth attendants have been trained and safe delivery kits have been made available everywhere. By the end of 2017 AD, Nepal targets to increase deliveries by trained persons to $95.0 \%$ and increase TT2 coverage in women to $90 \%$. Though there are certain constraints like underreporting of vaccination and cases, frequent shortage of vaccines, poor vaccine preventable disease surveillance in few districts and irregularity of immunisation sessions in some areas, we look forward to overcome these constraints and to see a tetanus free nation in a near future.

(a) Such as but not limited to, wounds contaminated with dirt, feces, soil or saliva. Puncture wounds; avulsions; and wounds resulting from missiles, crushing, burns or frost bite. (b) For children $<7$ years, the DPT or DT if pertusis vaccine is contra indicated is preferred to TT alone. For person $>7$ years, the Td is preferred to TT alone. (c) $\mathrm{TIG}=$ Tetanus immunoglobulin. TIG should be administered for tetanus prone wounds in HIV infected patients regardless of the history of tetanus immunisation. (d)Yes if $>10$ years since the last dose. (e) Yes if $>5$ years since the last dose. More frequent boosters are not needed and can accentuate adverse events.

Table 2: Summarised recommendation for the use of tetanus prophylaxis in routine wound management ${ }^{4}$.

\begin{tabular}{|l|c|c|c|c|}
\hline \multirow{2}{*}{$\begin{array}{l}\text { Prior Tetanus Vaccine } \\
\text { doses }\end{array}$} & \multicolumn{2}{|c|}{ Clean Minor Wounds } & \multicolumn{2}{|c|}{ Other Wounds $^{(\mathbf{a})}$} \\
\cline { 2 - 5 } & Td $^{(\mathbf{b})}$ & $\mathbf{T I G}^{(\mathbf{c})}$ & $\mathbf{T d}^{(\mathbf{b})}$ & TIG $^{(\mathbf{c})}$ \\
\hline uncertain or $<3$ & $\mathrm{Yes}$ & $\mathrm{No}$ & $\mathrm{Yes}$ & Yes \\
\hline$\geq 3$ & $\mathrm{No}^{(\mathrm{d})}$ & $\mathrm{No}$ & $\mathrm{No}^{(\mathrm{e})}$ & $\mathrm{No}$ \\
\hline
\end{tabular}

\section{References}

1. Finegold SM. Tetanus. In: Collier L, Balows A, Sussman M, editors. Topley and Wilson's Microbiology and Microbial Infections. 9th edition. London: Arnold; 1998. p. 693-717.

2. Bhatia R, Prabhakar S, Grover VK. Tetanus. Neurol India. 2002; 50: 398-407.

3. Department of Health Science, Ministry of Health and Population. Annual Report 2006 2007. Kathmandu: Ministry of Health and Population, Nepal. 2008.
4. Centre for Disease Control. Tetanus Surveillance - United States, 1998-2000. MMWR surveillance summaries [series on internet]. 2003;1-8. Available from: htpp://www.cdc.gov/MMWR/ PREVIEW/MMWRHTML/ss5203a1.htm

5. Aboud S, Lyamuya EF, Kristoffersen EK, Matre R. Tetanus Immunity among Pregnant Women Attending Antenatal Care in Dar es Salaam, Tanzania. African J Reproductive Health. 2002; $2: 87-93$. 
6. Park K. Park's Textbook of Preventive and Social medicine. $15^{\mathrm{t}} \mathrm{h}$ ed. India: M/s Banarsidas Bhanot Publishers; 1997. p. 237-40,346.

7. Department of Health Services, Kathmandu, Ministry of Health and Population, HMG of Nepal. Annual Report 2003\2004. Kathmandu: Ministry of Health and Population, Nepal. 2005.

8. Jha N. Review of Epidemiology of Diphtheria, Pertusis and Tetanus cases in Nepal. Final and Technical report. Dharan: BP Koirala Institute of Health Sciences; 2004.

9. Poudel P, Singh R, Raja S, Budhathoki S. Pediatric and neonatal tetanus: a hospital based study at eastern Nepal. Nepal Med Coll J. 2008; 10: 170-5.

10. Lau LG, Kong KO, Chew PH. A ten-year retrospective study of tetanus at a general hospital in Malaysia. Singapore Med J. 2001; 42: 346-50.

11. Cook TM, Protheoze RT, Handel JM. Tetanus: A review of literature. Br J Anaesth. 2001; 87: 477-87.

12. Prevots DR, Sutter RW. Tetanus. In: Wallance RB, Doebbeling BN, Last JM, editors. MaxcyRosenau-Last Public Health and preventive Medicine. 14th edition. USA: Appleton and Lange. 1998. p.102-5.

13. Patel JC, Mehta BC. Tetanus: study of 8697 cases. Indian J Med Sci. 1999; 53: 393-401.

14. Eman H, Adil M, Ayab M. Tetanus experience in a public hospital in western Saudi Arabia. Saudi Med J. 2003; 24: 1325-8.

15. Jeena PM, Coovadia HM, Gouws E. Risk factors for neonatal tetanus in KwaZulu-Natal. S Afr Med J. 1997; 87: 46-8.

16. Bleck TP. Tetanus: dealing with the continuing clinical challenge. J Crit Ill. 1987; 2: 41-52.

17. Brooks VB, Asanuma H. Pharmacological studies of recurrent cortical inhibition and facilitation. Am J Physiol. 1965; 208: 674-81.

18. Mellanby J, Green J. How does tetanus toxin work? Neuroscience. 1981; 6: 281-300.

19. Saltoglu N, Tosova Y, Midikli D, Burgut R, Dundar IH. Prognostic factors affecting deaths from adult tetanus. Clin Microbiol Infect Dis. 2004; 10: 229-33.

20. Volpe JJ. Neurology of the Newborn. 3rd edition. Philadelphia: W B Saunders; 1995. p. 759-61.

21. Wassay M, Khealani BA, Talati N, Shamsi R, Syed NA, Salahuddin N. Autonomic nervous system dysfunction predicts poor prognosis in patients with mild to moderate tetanus. BMC Neurol. 2005; 5: 1-4.

22. Juma M, Kaabi Al, Scrimgeour EM, Louon A, Bazdawi M, Riyami Al. Tetanus: a clinical review. Saudi Med J. 2001; 22: 606-9.

23. Jagoda A, Riggio S, Burguieres T. Cephalic tetanus: a case report and review of the literature. Am J Emerg Med. 1988; 6: 128-30.

24. Ablett JJL. Analysis and main experiences in 82 patients treated in the Leeds Tetanus Unit. In: Ellis M, ed. Symposium on Tetanus in Great Britain. Boston Spa, UK: National Lending Library.1967. p.1-10.

25. Illis LS, Taylor FM. Neurological and electroencephalographic sequelae of tetanus. Lancet. 1971; 1: 826-30.

26. Bwire R, Kawuma HJS. Hospital based study on neonatal tetanus, Buluba Hospital, 19851989. J Trop Med Hyg. 1992; 95: 62-6.

27. Arnon SS. Tetanus (Clostridium tetani). In: Behman RE, Kleigman RM, Jenson HB, editors. Nelson Textbook of Pediatrics. 17th edition. Philadelphia: Saunders; 2004. p. 951-3.

28. Borgeat A, Dessibourg C, Rochani M, Suter PM. Sedation by propofol in tetanus - is it a muscular relaxant? Intensive Care Med. 1991; 17: 427-9.

29. Engrand N, Vilain G, Rouamba A, Benhamou D. Value of intrathecal baclofen in the treatment of severe tetanus in the tropical milieu. Med Trop. 2000; 60: 385-8.

30. James MFM, Manson EDM. The use magnesium infusions in management of very severe tetanus. Intensive Care Med. 1985; 11: 5-12.

31. Buchanan N, Cane RD, Wolfson G, De Andrade M. Autonomic dysfunction in tetanus: the effects of a variety of therapeutic agents, with special reference to morphine. Intensive Care Med. 1979; 5: 65-8.

32. Dolar D. The use of continuous atropine infusion in the management of tetanus. Intensive Care Med. 1992;18:26-31.

33. Gregorakos L, Kerezoudi E, Dimopoulos G, Thomaides T. Management of blood pressure instability in severe tetanus: the use of clonidine. Intensive Care Med. 1997; 23: 893-5.

34. Farrar JJ, Yen LM, Cook T, Fairweather N, Binh N, Parry J et al. Tetanus. J Neurol Neurosurg Psy. 2000; 69: 292-301. 